\title{
The keratin cytoskeleton as a regulator of keratinocyte mechano-sensing
}

Ana Laly ${ }^{1}$, David Lee ${ }^{2}$ and John T. Connelly ${ }^{1}$

${ }^{1}$ Blizard Institute, Barts and the London School of Medicine and Dentistry, Queen Mary University of London

${ }^{2}$ School of Engineering and Materials Science, Queen Mary, University of London

\section{Background}

- Keratin cytoskeleton is an important cell shape and integrity regulator within the epidermis ${ }^{1}$

- When these filaments fail to assemble properly, keratinocytes become more fragile, which can lead to several skin blistering diseases ${ }^{2}$.

- It is still not clear how keratins can act as mediators for mechanotransduction.

- Polyacrylamide hydrogels can be used as an in vitro model to control matrix stiffness and further study cell-ECM interactions.

Aim: Dissecting the role of keratin cytoskeleton in cell mechanotransduction

I. Substrate stiffening promotes cell spreading
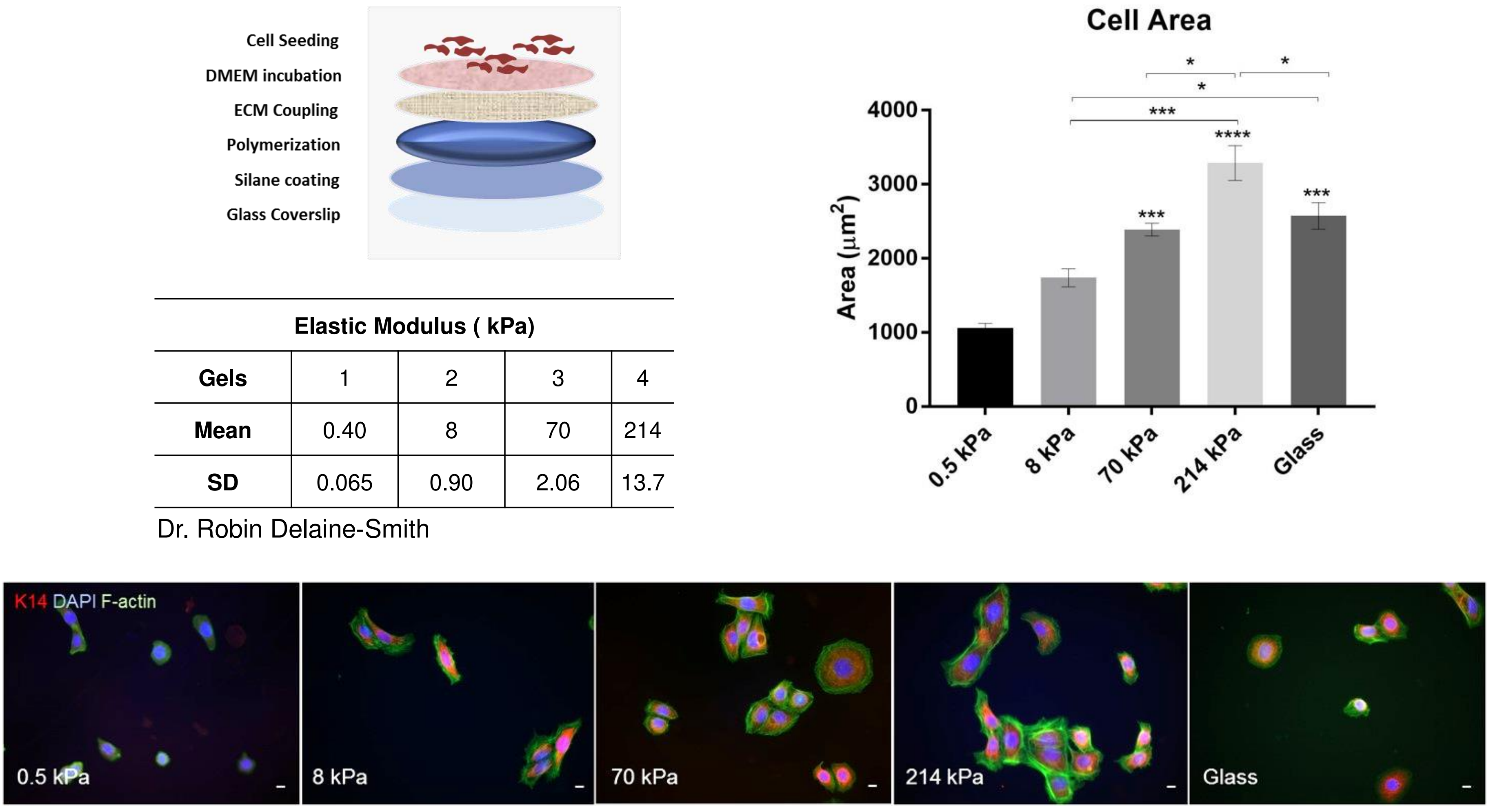

Figure 1 - HaCat cells seeded over polyacrylamide gels with different elastic modulus and glass. All substrates were coated with 50 $\mu \mathrm{g} / \mathrm{ml}$ type I Collagen in PBS. Cell area increases polyacrylamide with stiffness. Cell area data represent mean $\pm \mathrm{SEM}(\mathrm{N}=3$

\section{Substrate stiffening influences keratin 14 organisation}
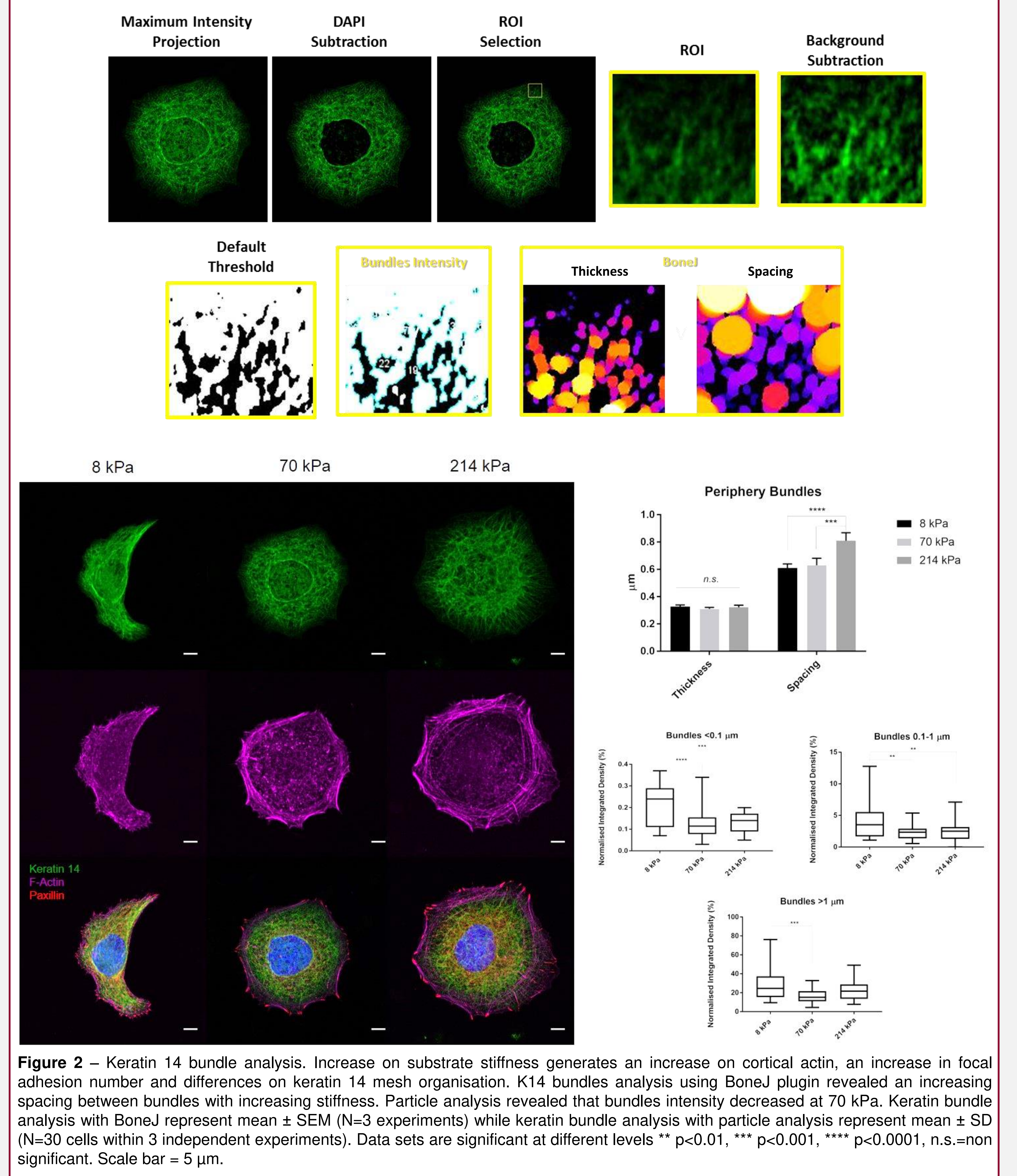

III. F-actin and Src affect stiffness-dependent cell spreading
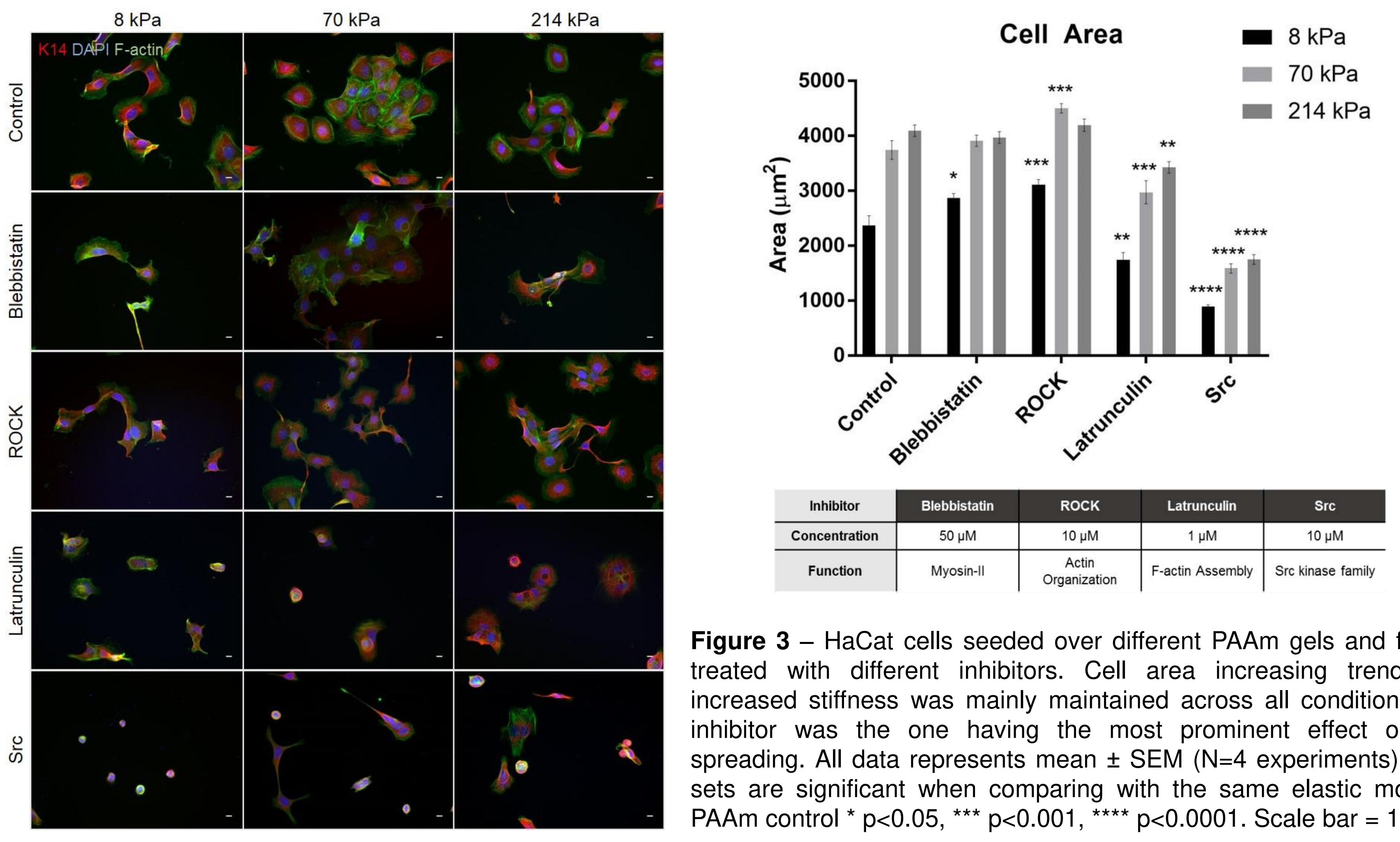

Figure 3 - HaCat cells seeded over different PAAm gels and further treated with different inhibitors. Cell area increasing trend with mhibitor was the one having the most prominent effect on preading. All data represents mean \pm SEM ( $N=4$ experiments) Dat sets are significant when comparing with the same elastic modulus
PAAm control ${ }^{*} p<0.05, * * * p<0.001,{ }^{* * * *} p<0.0001$. Scale bar $=10 \mu \mathrm{m}$.

IV. Plectin modulates stiffness-dependent cell spreading
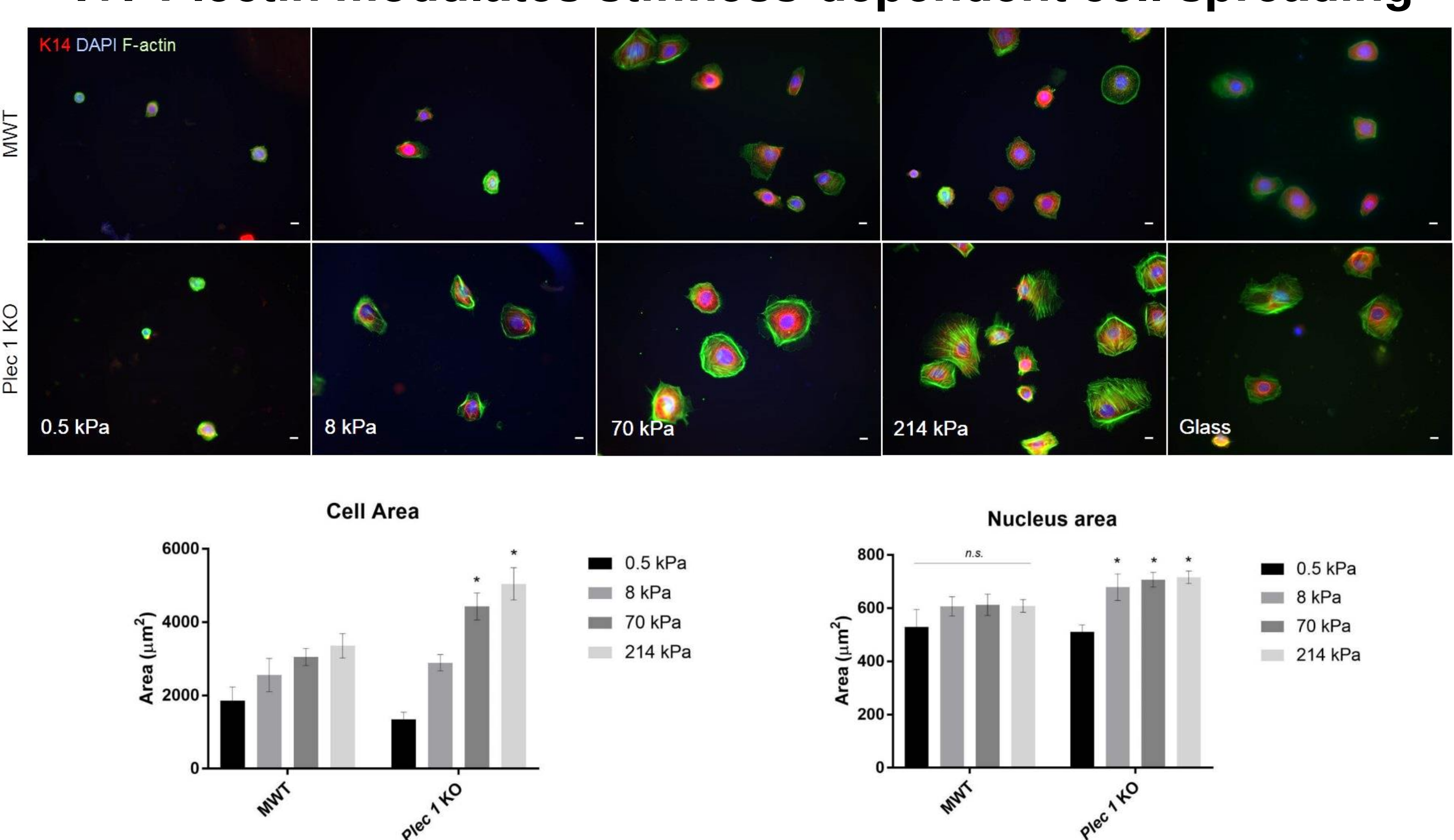

Figure 4 - Mouse keratinocytes wild type (MWT) and mouse keratinocyte plectin KO (Plec1 KO) cells seeded over polyacrylamide els with different elastic modulus and glass. All substrates were coated with $50 \mathrm{mg} / \mathrm{ml}$ type I Collagen in PBS. Plectin KO cells area showed an increase at 7 and $214 \mathrm{KPa}$ PAAm gels when comparing Wh $M$ WT. Nucleus area showed to be increased on Plectin KO significant when comparing with MWT cell area at the same elastic modulus PAAm * $\mathrm{p}<0.05$, n.s. =non significant. Scale bar $=10 \mu \mathrm{m}$.

V. Substrate stiffness and plectin regulate EGFR signalling
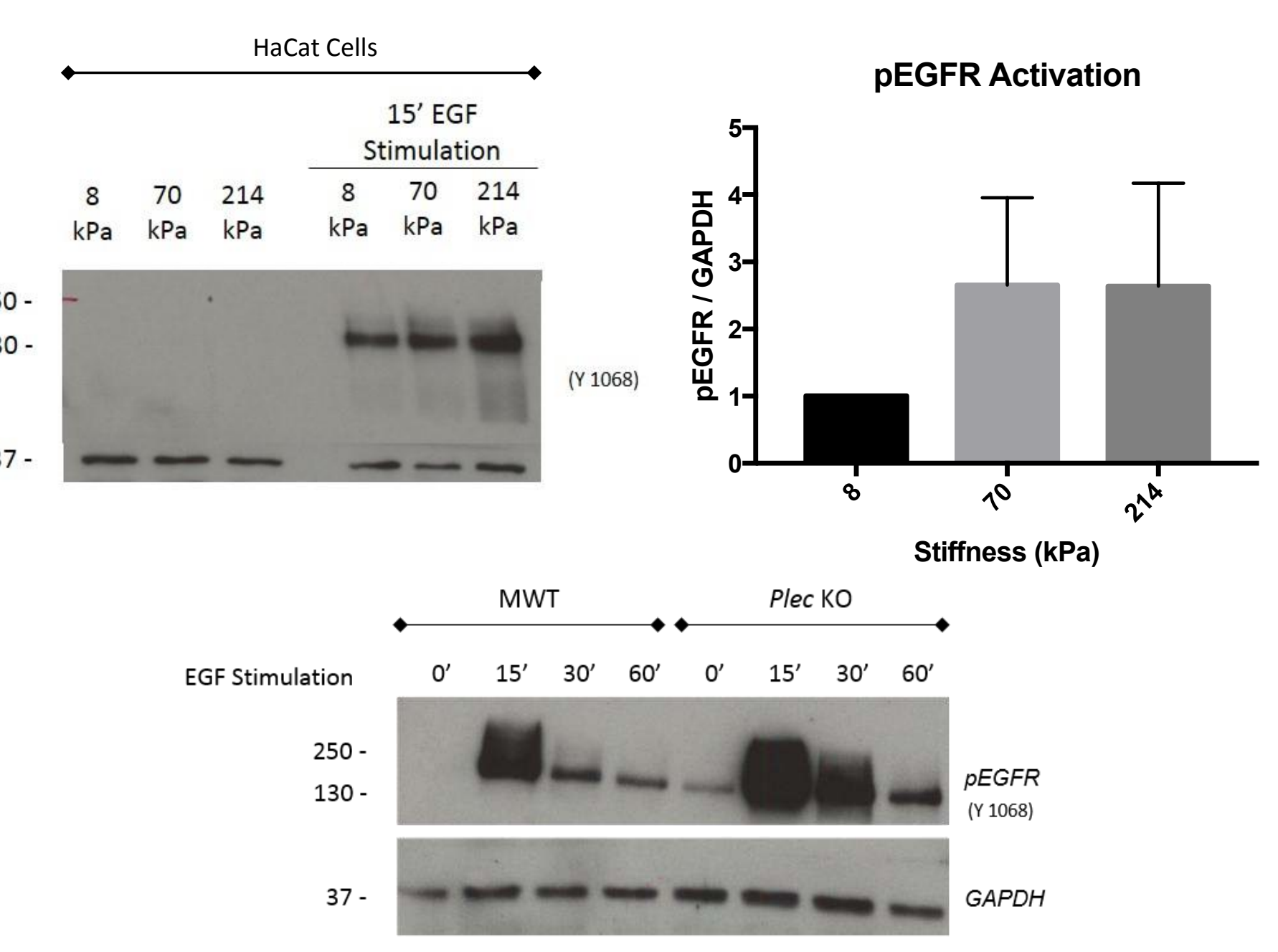

Figure 5 - Phosphorylated EGFR expression with and without EGF for 15 minutes when seeded over PAAm gels with different stiffnesses. pEGFR increased both after stimulation and with stiffness. Mouse keratinocytes (Wild-type and Plec1 KO) pEGFR signalling levels were also assessed after EGF stimulation increase on Plec1 KO when comparing with MWT different timepoints. Moreover, the peak of $p E G F R$ levels was
VI. Substrate stiffness and keratins regulate Lamin A levels
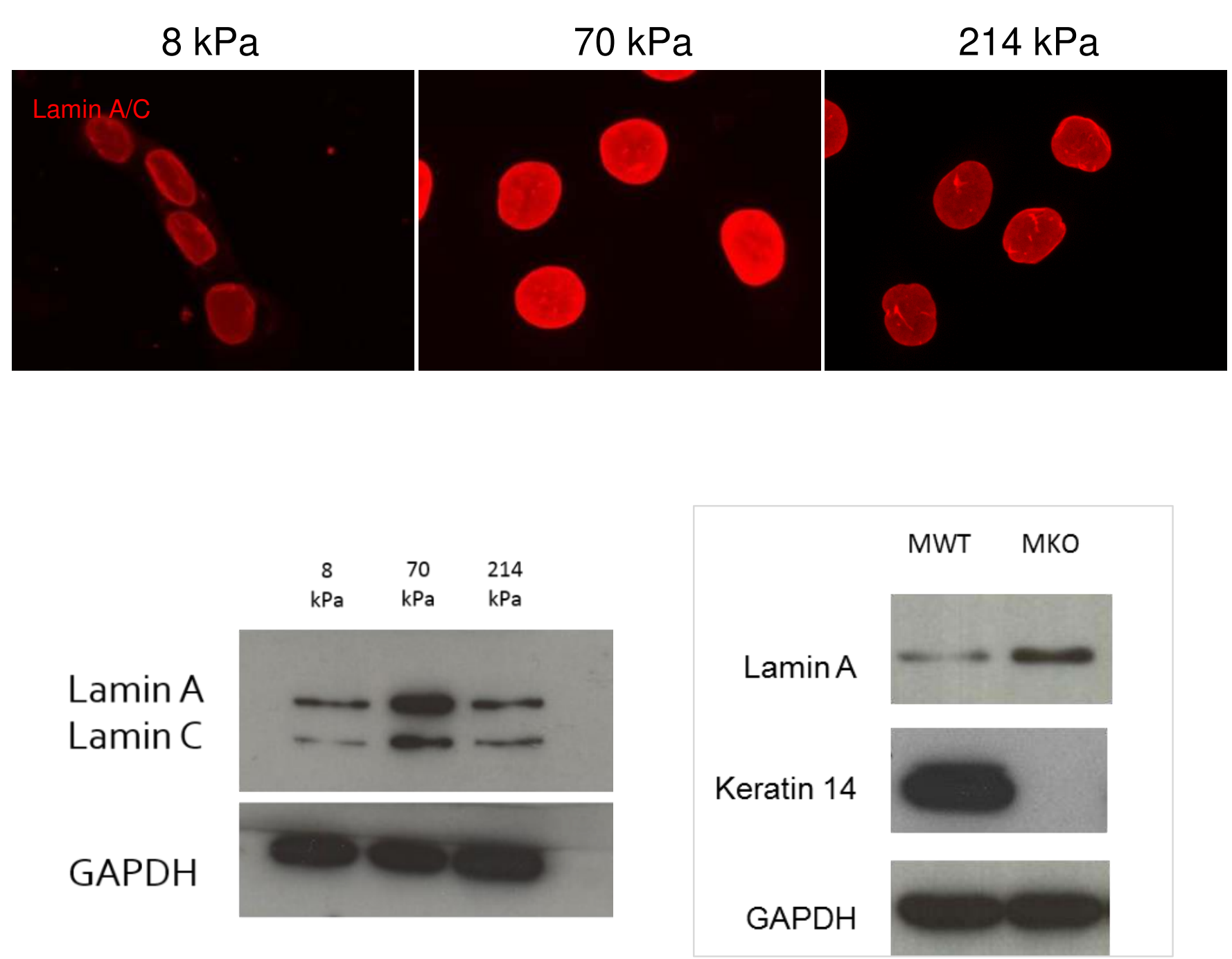

VII. Conclusion \& Future work

- Substrate stiffness regulates cell spreading.

- Cell contractility elements have different roles in cellular mechanotransduction.

- Keratin 14 bundles spacing and intensity changes in response to substrate stiffness.
- Substrate stiffness can regulate EGFR phosphorylation

- The cytolinker protein Plectin has an active role in cell spreading and EGFR phosphorylation

- Substrate stiffness regulates Lamin A/C expression and depends on type I keratins 